IFUSP-P-1211

\title{
Super-Poincaré Invariant Koba-Nielsen Formulas for the Superstring
}

\author{
Nathan Berkovits \\ Dept. de Física Matemática, Univ. de São Paulo \\ CP 20516, São Paulo, SP 01498, BRASIL \\ and \\ IMECC, Univ. de Campinas \\ CP 1170, Campinas, SP 13100, BRASIL \\ e-mail: nberkovi@snfma2.if.usp.br
}

\begin{abstract}
The new spacetime-supersymmetric description of the superstring is used to compute tree-level scattering amplitudes for an arbitrary number of massless four-dimensional states. The resulting Koba-Nielsen formula is manifestly $\mathrm{SO}(3,1)$ super-Poincaré invariant and is easily generalized to scattering in the presence of a D-brane.
\end{abstract}

April 1996 


\section{Introduction}

Before the work of this author, all spacetime-supersymmetric calculations of superstring amplitudes used the light-cone Green-Schwarz formalism, which requires non-trivial operators at the light-cone interaction points. [1] [2] [3] Because of this complication, only the four-point massless tree and one-loop amplitudes had been explicitly computed in a spacetime-supersymmetric manner.

Over the last few years, this author has developed an alternative spacetimesupersymmetric description of the superstring which has the advantage of being conformally invariant, and therefore not requiring non-trivial interaction-point operators. [5]. The new formalism is manifestly $\mathrm{SO}(3,1)$ super-Poincaré invariant and is suitable for any compactification to four dimensions which preserves $\mathrm{N}=14 \mathrm{D}$ supersymmetry. Furthermore, the new formalism contains critical $\mathrm{N}=2$ worldsheet superconformal invariance, and is related by a field redefinition to the $\mathrm{N}=2$ embedding of the $\mathrm{N}=1 \mathrm{RNS}$ superstring. [6]

Together with Vafa, a simple "topological" method was developed for calculating scattering amplitudes in this formalism. [7] (An easily accessible review of this method can be found in reference [8]) In this letter, the topological method will be used to explicitly compute tree-level scattering amplitudes for an arbitrary number of four-dimensional massless states. The result can be expressed as a Koba-Nielsen formula, and is manifestly $\mathrm{SO}(3,1)$ super-Poincaré invariant.

It is straightforward to generalize the formula for scattering in the presence of a Dbrane. This result should be useful for D-brane analysis since, unlike computations in the RNS formalism, [9] it is manifestly spacetime-supersymmetric, and therefore includes both NS-NS and R-R couplings.

1 Although reference [3] contains explicit expressions for four-point multiloop amplitudes, these expressions contain unphysical divergences when interaction points coincide. It has not yet been determined how these expressions are affected by removing the divergences with contact terms. Also, Mandelstam has constructed an N-point tree amplitude which has manifest $\mathrm{SU}(4) \times \mathrm{U}(1)$ super-Poincaré invariance. [4] However the structure of his amplitude was obtained indirectly by unitarity arguments, rather than by explicit computations. It would be interesting to compare his amplitude with the Koba-Nielsen formula in this letter. 


\section{Review}

As shown in reference [7], the topological prescription for computing the tree-level amplitude for $\mathrm{N}$ open superstring states in any $\mathrm{c}=6 \mathrm{~N}=2$ string theory is

$$
\mathcal{A}=<V_{1}\left(z_{1}\right) G^{+}\left(V_{2}\left(z_{2}\right)\right) \tilde{G}^{+}\left(V_{3}\left(z_{3}\right)\right) \prod_{r=4}^{N} \int d z_{r} G^{-}\left(G^{+}\left(V_{r}\left(z_{r}\right)\right)\right)>
$$

where $V(z)$ is a $\mathrm{U}(1)$-neutral $\mathrm{N}=2$ primary field of weight $0,\left[T, G^{+}, G^{-}, J\right]$ are the twisted $\mathrm{c}=6 \mathrm{~N}=2$ generators (after twisting, $G^{+}$has weight 1 and $G^{-}$has weight 2 ), $G^{ \pm}(Y)$ means the contour integral of $G^{ \pm}$around $Y, \tilde{G}^{+}=G^{-}\left(e^{i H}\right)$, and $J=\partial H$. The Chan-Paton factors will be supressed throughout this letter.

When (2.1)is applied to the $\mathrm{N}=2$ embedding of the $\mathrm{N}=1$ RNS superstring, it reproduces the standard RNS prescription in the "large" Hilbert space:

$$
\mathcal{A}=<\xi\left(z_{1}\right) V_{1}^{R N S}\left(z_{1}\right) Z V_{2}^{R N S}\left(z_{2}\right) V_{3}^{R N S}\left(z_{3}\right) \prod_{r=4}^{N} \int d z_{r}\left\{b, Z V_{r}^{R N S}\left(z_{r}\right)\right\}>
$$

since in the $\mathrm{N}=2$ embedding, $V=\xi V^{R N S}, G^{+}=j_{B R S T}, G^{-}=b$, and $\tilde{G}^{+}=\eta$. (Note that $\beta=\partial \xi e^{-\phi}$ and $\gamma=\eta e^{\phi}$ are the bosonic RNS ghosts, $Z=\{Q, \xi\}$ is the RNS picturechanging operator, $V^{R N S}$ is in the -1 picture, and $Z V^{R N S}=\left\{Q, \xi V^{R N S}\right\}$ is in the 0 picture). So $G^{+}(V)=Q\left(\xi V^{R N S}\right)=Z V^{R N S}$ and $\tilde{G}^{+}(V)=V^{R N S}$.

In the new spacetime-supersymmetric description of the superstring, a field redefinition is used to write the generators of the $\mathrm{N}=2$ embedding in terms of spacetimesupersymmetric variables. [5] In terms of the four-dimensional supersymmetric variables $\left[x^{m}, \theta^{\alpha}, \bar{\theta}^{\dot{\alpha}}, p_{\alpha}, \bar{p}_{\dot{\alpha}}, \rho\right]$ and the compactification-dependent variables, the twisted $\mathrm{c}=6 \mathrm{~N}=2$ generators are:

$$
\begin{gathered}
T=\frac{1}{2} \partial x^{m} \partial x_{m}+p_{\alpha} \partial \theta^{\alpha}+\bar{p}_{\dot{\alpha}} \partial \bar{\theta}^{\dot{\alpha}}+\frac{1}{2}\left(\partial \rho \partial \rho+i \partial^{2} \rho\right)+T_{C}, \\
G^{+}=e^{i \rho}(d)^{2}+G_{C}^{+}, \quad G^{-}=e^{-i \rho}(\bar{d})^{2}+G_{C}^{-}, \quad J=-\partial \rho+J_{C},
\end{gathered}
$$

where $m$ takes values 0 to $3, \alpha$ and $\dot{\alpha}$ take values 1 or $2, p_{\alpha}$ and $\bar{p}_{\dot{\alpha}}$ are conjugates to $\theta^{\alpha}$ and $\bar{\theta}^{\dot{\alpha}}, \rho$ is a chiral boson,

$$
\begin{aligned}
& d_{\alpha}=p_{\alpha}+\frac{i}{2} \bar{\theta}^{\dot{\alpha}} \partial x_{\alpha \dot{\alpha}}-\frac{1}{8}(\bar{\theta})^{2} \partial \theta_{\alpha}+\frac{1}{16} \theta_{\alpha} \partial(\bar{\theta})^{2}, \\
& \bar{d}_{\dot{\alpha}}=\bar{p}_{\dot{\alpha}}+\frac{i}{2} \theta^{\alpha} \partial x_{\alpha \dot{\alpha}}-\frac{1}{8}(\theta)^{2} \partial \bar{\theta}_{\dot{\alpha}}+\frac{1}{16} \bar{\theta}_{\dot{\alpha}} \partial(\theta)^{2},
\end{aligned}
$$


$(d)^{2}$ means $\frac{1}{2} \epsilon^{\alpha \beta} d_{\alpha} d_{\beta}$, and $\left[T_{C}, G_{C}^{+}, G_{C}^{-}, J_{C}\right]$ are the twisted $\mathrm{c}=9 \mathrm{~N}=2$ generators of the superconformal field theory used to describe the compactification manifold.

The action in conformal gauge for these fields is

$$
\begin{gathered}
\int d z d \bar{z}\left(\frac{1}{2} \partial x^{m} \bar{\partial} x_{m}+p_{L \alpha} \bar{\partial} \theta_{L}^{\alpha}+\bar{p}_{L \dot{\alpha}} \bar{\partial} \bar{\theta}_{L}^{\dot{\alpha}}+\frac{1}{2} \partial \rho_{L} \bar{\partial} \rho_{L}\right. \\
\left.+p_{R \alpha} \partial \theta_{R}^{\alpha}+\bar{p}_{R \dot{\alpha}} \partial \bar{\theta}_{R}^{\dot{\alpha}}+\frac{1}{2} \partial \rho_{R} \bar{\partial} \rho_{R}\right)+S_{C}
\end{gathered}
$$

where $S_{C}$ is the action for the compactification-dependent variables. When the $R / L$ index has been suppressed (as in (2.3)), we shall always mean left-moving. Note that $z$ versus $\bar{z}$ is correlated with $L$ versus $R$, and not with $\theta$ versus $\bar{\theta}$.

The free-field OPE's of the four-dimensional variables are

$$
\begin{gathered}
x^{m}(y) x^{n}(z) \rightarrow \eta^{m n} \log |y-z|^{2}, \\
\rho_{L}(y) \rho_{L}(z) \rightarrow \log (y-z), \quad \rho_{R}(y) \rho_{R}(z) \rightarrow \log (\bar{y}-\bar{z}), \\
p_{L \alpha}(y) \theta_{L}^{\beta}(z) \rightarrow \frac{\delta_{\alpha}^{\beta}}{y-z}, \quad \bar{p}_{L \dot{\alpha}}(y) \bar{\theta}_{L}^{\dot{\beta}}(z) \rightarrow \frac{\delta_{\dot{\alpha}}^{\dot{\beta}}}{y-z}, \\
p_{R \alpha}(y) \theta_{R}^{\beta}(z) \rightarrow \frac{\delta_{\alpha}^{\beta}}{\bar{y}-\bar{z}}, \quad \bar{p}_{R \dot{\alpha}}(y) \bar{\theta}_{R}^{\dot{\beta}}(z) \rightarrow \frac{\delta_{\dot{\alpha}}^{\dot{\beta}}}{\bar{y}-\bar{z}} .
\end{gathered}
$$

Note that the chiral boson $\rho$ can not be fermionized since $e^{i \rho(y)} e^{-i \rho(z)} \rightarrow(y-z)$. It has the same behavior as the negative-energy field $\phi$ that appears when bosonizing the RNS ghosts. After twisting, the background charge is defined for open superstring tree amplitudes by

$$
<(\theta)^{2}(\bar{\theta})^{2} e^{-i \rho+i H_{C}}>=1
$$

where $J_{C}=\partial H_{C}$.

As was shown by Siegel, 10 d $d_{\alpha}$ and $\bar{d}_{\dot{\alpha}}$ satisfy the OPE that $d_{\alpha}(y) d_{\beta}(z)$ is regular,

$$
\begin{gathered}
d_{\alpha}(y) \bar{d}_{\dot{\alpha}}(z) \rightarrow i \frac{\Pi_{\alpha \dot{\alpha}}}{y-z} \\
d_{\alpha}(y) \Pi^{m}(z) \rightarrow-i \frac{\sigma_{\alpha \dot{\alpha}}^{m} \partial \bar{\theta}^{\dot{\alpha}}}{y-z}, \quad \bar{d}_{\dot{\alpha}}(y) \Pi^{m}(z) \rightarrow-i \frac{\sigma_{\alpha \dot{\alpha}}^{m} \partial \theta^{\alpha}}{y-z},
\end{gathered}
$$

where

$$
\Pi_{\alpha \dot{\alpha}}=\sigma_{\alpha \dot{\alpha}}^{m} \partial x_{m}-\frac{i}{2} \theta_{\alpha} \partial \bar{\theta}_{\dot{\alpha}}-\frac{i}{2} \bar{\theta}_{\dot{\alpha}} \partial \theta_{\alpha}
$$


The advantage of working with the variables $d_{\alpha}$ and $\Pi_{m}$ is that they commute with the spacetime supersymmetry generators,

$$
q_{\alpha}=\oint d z\left[p_{\alpha}-\frac{i}{2} \bar{\theta}^{\dot{\alpha}} \partial x_{\alpha \dot{\alpha}}-\frac{1}{16}(\bar{\theta})^{2} \partial \theta_{\alpha}\right], \quad \bar{q}_{\dot{\alpha}}=\oint d z\left[\bar{p}_{\dot{\alpha}}-\frac{i}{2} \theta^{\alpha} \partial x_{\alpha \dot{\alpha}}-\frac{1}{16}(\theta)^{2} \partial \bar{\theta}_{\dot{\alpha}}\right] .
$$

\section{Koba-Nielsen formula for open superstrings}

For massless states of the open superstring which are independent of the compactification, the vertex operator $V$ only depends on the zero modes of $x^{m}, \theta^{\alpha}$ and $\bar{\theta}^{\dot{\alpha}}$. It can therefore be represented by the superfield $V(x, \theta, \bar{\theta})$, which is the standard $\mathrm{N}=14 \mathrm{D}$ super-Yang-Mills prepotential (e.g. the $\theta \bar{\theta}$ component of $V$ is the gluon gauge field $A^{m}$, and the $\theta(\bar{\theta})^{2}$ and $(\theta)^{2} \bar{\theta}$ components of $V$ are the gluino fields $\xi^{\alpha}$ and $\left.\bar{\xi}^{\dot{\alpha}}\right)$.

The condition of being $\mathrm{N}=2$ primary implies that

$$
(D)^{2} V=(\bar{D})^{2} V=\partial_{m} \partial^{m} V=0
$$

where $D_{\alpha}=\partial_{\theta^{\alpha}}+\frac{i}{2} \bar{\theta}^{\dot{\alpha}} \sigma_{\alpha \dot{\alpha}}^{m} \partial_{m}$ and $\bar{D}_{\dot{\alpha}}=\partial_{\bar{\theta}^{\dot{\alpha}}}+\frac{i}{2} \theta^{\alpha} \sigma_{\alpha \dot{\alpha}}^{m} \partial_{m}$ are the covariant fermionic derivatives. Note that $(D)^{2} V=(\bar{D})^{2} V=0$ is the standard supersymmetric generalization of the Lorentz gauge condition $\partial_{m} A^{m}=0$.

Using the free-field OPE's of (2.5), it is straightforward to compute that

$$
\begin{gathered}
\int d z G^{-}\left(G^{+}(V)\right)=\int d z\left(d^{\alpha}(\bar{D})^{2} D_{\alpha} V+\bar{d}^{\dot{\alpha}}(D)^{2} \bar{D}_{\dot{\alpha}} V\right. \\
\left.+\partial \theta^{\alpha} D_{\alpha} V-\partial \bar{\theta}^{\dot{\alpha}} \bar{D}_{\dot{\alpha}} V-i \Pi^{\alpha \dot{\alpha}}\left[D_{\alpha}, \bar{D}_{\dot{\alpha}}\right] V\right) .
\end{gathered}
$$

Plugging into (2.1),

$$
\begin{gathered}
\mathcal{A}=<V_{1}\left(z_{1}\right) e^{i \rho} d^{\alpha}\left(z_{2}\right) D_{\alpha} V_{2}\left(z_{2}\right) e^{-2 i \rho+H_{C}} \bar{d}^{\dot{\alpha}}\left(z_{3}\right) \bar{D}_{\dot{\alpha}} V_{3}\left(z_{3}\right) \\
\prod_{r=4}^{N} \int d z_{r}\left(d^{\alpha}\left(z_{r}\right)(\bar{D})^{2} D_{\alpha} V_{r}\left(z_{r}\right)+\bar{d}^{\dot{\alpha}}\left(z_{r}\right)(D)^{2} \bar{D}_{\dot{\alpha}} V_{r}\left(z_{r}\right)\right. \\
\left.+\partial \theta^{\alpha}\left(z_{r}\right) D_{\alpha} V_{r}\left(z_{r}\right)-\partial \bar{\theta}^{\dot{\alpha}}\left(z_{r}\right) \bar{D}_{\dot{\alpha}} V_{r}\left(z_{r}\right)-i \Pi^{\alpha \dot{\alpha}}\left(z_{r}\right)\left[D_{\alpha}, \bar{D}_{\dot{\alpha}}\right] V_{r}\left(z_{r}\right)\right)> \\
=\left(\left.\prod_{r=1}^{N} \frac{\partial}{\partial \epsilon_{r}}\right|_{\epsilon_{r}=0}\right) \prod_{s=4}^{N} \int d z_{s} \epsilon_{1} \\
<\exp \left(\sum_{r=1}^{N} \epsilon_{r}\left[d_{\alpha}\left(z_{r}\right) w_{r}^{\alpha}+\bar{d}_{\dot{\alpha}}\left(z_{r}\right) \bar{w}_{r}^{\dot{\alpha}}+\partial \theta_{\alpha}\left(z_{r}\right) a_{r}^{\alpha}+\partial \bar{\theta}_{\dot{\alpha}}\left(z_{r}\right) \bar{a}_{r}^{\dot{\alpha}}+\Pi_{\alpha \dot{\alpha}}\left(z_{r}\right) a_{r}^{\alpha \dot{\alpha}}\right]\right)
\end{gathered}
$$




$$
e^{i \rho\left(z_{2}\right)} e^{-2 i \rho\left(z_{3}\right)+i H_{C}\left(z_{3}\right)} \prod_{r=1}^{N} V_{r}\left(z_{r}\right)>
$$

where

$$
\begin{gathered}
w_{2}^{\alpha}=D_{2}^{\alpha}, \quad \bar{w}_{2}^{\dot{\alpha}}=a_{2}^{\alpha}=\bar{a}_{2}^{\dot{\alpha}}=a_{2}^{\alpha \dot{\alpha}}=0, \\
\bar{w}_{3}^{\dot{\alpha}}=\bar{D}_{3}^{\dot{\alpha}}, \quad w_{3}^{\alpha}=a_{3}^{\alpha}=\bar{a}_{3}^{\dot{\alpha}}=a_{3}^{\alpha \dot{\alpha}}=0 \\
\bar{w}_{r}^{\dot{\alpha}}=\left(D_{r}\right)^{2} \bar{D}_{r}^{\dot{\alpha}}, w_{r}^{\alpha}=\left(\bar{D}_{r}\right)^{2} D_{r}^{\alpha}, a_{r}^{\alpha}=D_{r}^{\alpha}, \bar{a}_{r}^{\dot{\alpha}}=-\bar{D}_{r}^{\dot{\alpha}}, a_{r}^{\alpha \dot{\alpha}}=-i\left[D_{r}^{\alpha}, \bar{D}_{r}^{\dot{\alpha}}\right]
\end{gathered}
$$

for $r>3$, and $D_{r}^{\alpha}$ and $\bar{D}_{r}^{\dot{\alpha}}$ are fermionic derivatives which act only on $V_{r}\left(z_{r}\right)$. For example,

$$
D_{2}^{\alpha} \prod_{r=1}^{N} V_{r}\left(z_{r}\right)=V_{1}\left(z_{1}\right) D^{\alpha} V_{2}\left(z_{2}\right) \prod_{r=3}^{N} V_{r}\left(z_{r}\right) .
$$

Using the fact that $\left\langle d^{\alpha}(z) \ldots\right\rangle=\sum_{s}\left\langle\frac{f_{s}^{\alpha}\left(z_{s}\right)}{z-z_{s}}\right\rangle$, where $f_{s}$ is the residue of the pole at $z=z_{s}$, one can use the OPE's of (2.7)to write

$$
\begin{gathered}
\mathcal{A}=\left(\left.\prod_{r=1}^{N} \frac{\partial}{\partial \epsilon_{r}}\right|_{\epsilon_{r}=0}\right) \prod_{s=4}^{N} \int d z_{s} \epsilon_{1} \\
<\exp \left(\sum_{r, s} \epsilon_{r}\left[\frac{D_{s \alpha}}{z_{r}-z_{s}}+\epsilon_{s}\left(\frac{i \Pi_{\alpha \dot{\alpha}}\left(z_{s}\right) \bar{w}_{s}^{\dot{\alpha}}-i \partial \bar{\theta}_{\dot{\alpha}}\left(z_{s}\right) a_{s \alpha \dot{\alpha}}}{z_{r}-z_{s}}+\frac{a_{s \alpha}}{\left(z_{r}-z_{s}\right)^{2}}\right)\right] w_{r}^{\alpha}\right. \\
+\frac{1}{2} \sum_{r, s, t} \epsilon_{r} \epsilon_{s} \epsilon_{t} \frac{\partial \bar{\theta}_{\dot{\alpha}}\left(z_{s}\right) \bar{w}_{s}^{\dot{\alpha}} w_{t \alpha} w_{r}^{\alpha}}{\left(z_{r}-z_{s}\right)\left(z_{t}-z_{s}\right)} \\
\left.+\sum_{r} \epsilon_{r}\left[\bar{d}^{\dot{\alpha}}\left(z_{r}\right) \bar{w}_{r}^{\dot{\alpha}}+\partial \bar{\theta}_{\dot{\alpha}}\left(z_{r}\right) \bar{a}_{r}^{\dot{\alpha}}+\Pi_{\alpha \dot{\alpha}}\left(z_{r}\right) a_{r}^{\alpha \dot{\alpha}}\right]\right) \\
e^{i \rho\left(z_{2}\right)} e^{-2 i \rho\left(z_{3}\right)+H_{C}\left(z_{3}\right)} \prod_{r=1}^{N} V_{r}\left(z_{r}\right)>
\end{gathered}
$$

where $D_{r}^{\alpha}$ is always ordered to the left of $w_{r}$ and $a_{r}$. The term proportional to $\epsilon_{r} \epsilon_{s} \epsilon_{t}$ comes from the pole of $d^{\alpha}\left(z_{t}\right)$ with the residue of the pole of $d^{\beta}\left(z_{r}\right)$ at $z_{s}$.

One can similarly use the OPE's for $\bar{d}^{\dot{\alpha}}$ to write

$$
\begin{gathered}
\mathcal{A}=\left(\left.\prod_{r=1}^{N} \frac{\partial}{\partial \epsilon_{r}}\right|_{\epsilon_{r}=0}\right) \prod_{s=4}^{N} \int d z_{s} \epsilon_{1} \\
<\exp \left(\sum_{r, s} \epsilon_{r}\left[\frac{D_{s \alpha} w_{r}^{\alpha}+\bar{D}_{s \dot{\alpha}} \bar{w}_{r}^{\dot{\alpha}}}{z_{r}-z_{s}}+\epsilon_{s}\left(\frac{i \Pi_{\alpha \dot{\alpha}}\left(z_{s}\right) \bar{w}_{s}^{\dot{\alpha}} w_{r}^{\alpha}}{z_{r}-z_{s}}+\frac{a_{s \alpha} w_{r}^{\alpha}+\bar{a}_{s \dot{\alpha}} \bar{w}_{r}^{\dot{\alpha}}}{\left(z_{r}-z_{s}\right)^{2}}\right)\right]\right.
\end{gathered}
$$




$$
\begin{gathered}
-i \sum_{r, s, t} \epsilon_{r} \epsilon_{s} \epsilon_{t} \frac{a_{t \alpha \dot{\alpha}} w_{s}^{\alpha} \bar{w}_{r}^{\dot{\alpha}}}{\left(z_{r}-z_{t}\right)^{2}\left(z_{s}-z_{t}\right)} \\
\left.+\frac{1}{2} \sum_{r, s, t, u} \epsilon_{r} \epsilon_{s} \epsilon_{t} \epsilon_{u} \frac{\bar{w}_{t \dot{\alpha}} w_{u \alpha} w_{s}^{\alpha} \bar{w}_{r}^{\dot{\alpha}}}{\left(z_{r}-z_{t}\right)^{2}\left(z_{s}-z_{t}\right)\left(z_{u}-z_{t}\right)}+\sum_{r} \epsilon_{r} \Pi_{\alpha \dot{\alpha}}\left(z_{r}\right) a_{r}^{\alpha \dot{\alpha}}\right) \\
e^{i \rho\left(z_{2}\right)} e^{-2 i \rho\left(z_{3}\right)+i H_{C}\left(z_{3}\right)} \prod_{r=1}^{N} V_{r}\left(z_{r}\right)>
\end{gathered}
$$

where $\bar{D}_{r}^{\dot{\alpha}}$ is always ordered to the left of $D_{r}^{\alpha}$, which is always ordered to the left of $w_{r}$ and $a_{r}$.

Finally, performing the correlation function over the $x$ 's and $\rho$, and using the normalization of (2.6), one obtains

$$
\begin{gathered}
\mathcal{A}=\left(\left.\prod_{r=1}^{N} \frac{\partial}{\partial \epsilon_{r}}\right|_{\epsilon_{r}=0}\right) \prod_{s=4}^{N} \int d z_{s} \epsilon_{1} \\
\int d^{2} \theta_{0} d^{2} \bar{\theta}_{0}: \exp \left(\sum_{r, s} \epsilon_{r}\left[\frac{D_{s \alpha} w_{r}^{\alpha}+\bar{D}_{s \dot{\alpha}} \bar{w}_{r}^{\dot{\alpha}}+2 k_{s m} a_{r}^{m}}{z_{r}-z_{s}}+\epsilon_{s} \frac{a_{s \alpha} w_{r}^{\alpha}+\bar{a}_{s \dot{\alpha}} \bar{w}_{r}^{\dot{\alpha}}}{\left(z_{r}-z_{s}\right)^{2}}\right]\right. \\
+i \sum_{r, s, t} \epsilon_{r} \epsilon_{s}\left(\frac{2 w_{r}^{\alpha} \bar{w}_{s}^{\dot{\alpha}} k_{t \alpha \dot{\alpha}}}{\left(z_{s}-z_{r}\right)\left(z_{s}-z_{t}\right)}+\epsilon_{t} \frac{w_{r}^{\alpha} \bar{w}_{s}^{\dot{\alpha}} a_{t \alpha \dot{\alpha}}}{\left(z_{s}-z_{t}\right)\left(z_{r}-z_{t}\right)\left(z_{r}-z_{s}\right)}\right) \\
\left.+\frac{1}{4} \sum_{r, s, t, u} \epsilon_{r} \epsilon_{s} \epsilon_{t} \epsilon_{u} \frac{\bar{w}_{s \dot{\alpha}} \bar{w}_{u}^{\dot{\alpha}} w_{t \alpha} w_{r}^{\alpha}}{\left(z_{r}-z_{s}\right)\left(z_{t}-z_{u}\right)\left(z_{r}-z_{u}\right)\left(z_{t}-z_{s}\right)}\right): \\
\left(z_{2}-z_{3}\right)^{2} \prod_{r, s}\left(z_{r}-z_{s}\right)^{4 k_{r}^{m} k_{s m}} \prod_{r} V_{r}\left(z_{r}\right)
\end{gathered}
$$

where $k_{r}^{m}$ is the momentum of $V_{r},: \exp ():$ is to remind that $\bar{D}_{r}^{\dot{\alpha}}$ is always ordered to the left of $D_{r}^{\alpha}$ which is always ordered to the left of $w_{r}$ and $a_{r}$, and $\int d^{2} \theta_{0} d^{2} \bar{\theta}_{0}$ means to set $\theta^{\alpha}\left(z_{r}\right)=\theta_{0}^{\alpha}$ and $\bar{\theta}^{\dot{\alpha}}\left(z_{r}\right)=\bar{\theta}_{0}^{\dot{\alpha}}$ for all $r$ and to integrate over $\theta_{0}^{\alpha}$ and $\bar{\theta}_{0}^{\dot{\alpha}}$.

It is straightforward to check that $\mathcal{A}$ is gauge-invariant since under the transformation $\delta V_{p}=(\bar{D})^{2} \Phi_{p}\left(\right.$ which implies $a_{p}^{\dot{\alpha}}=D_{p}^{\dot{\alpha}}, a_{p}^{m}=k_{p}^{m}$, and $\left.\bar{a}_{p}^{\dot{\alpha}}=w_{p}^{\alpha}=\bar{w}_{p}^{\dot{\alpha}}=0\right)$

$$
\begin{gathered}
\delta \mathcal{A}=\left(\left.\prod_{r=1}^{N} \frac{\partial}{\partial \epsilon_{r}}\right|_{\epsilon_{r}=0}\right) \prod_{s=4}^{N} \int d z_{s} \epsilon_{1} \epsilon_{p} \frac{\partial}{\partial z_{p}} \\
\int d^{2} \theta_{0} d^{2} \bar{\theta}_{0}: \exp F:\left(z_{2}-z_{3}\right)^{2} \prod_{r, s}\left(z_{r}-z_{s}\right)^{k_{r}^{m} k_{s m}}\left(\bar{D}_{p}\right)^{2} \Phi_{p} \prod_{r \neq p} V_{r}\left(z_{r}\right)
\end{gathered}
$$

where $F$ is the object in the exponential of (3.4). 


\section{Koba-Nielsen formula for closed superstrings}

For the closed superstring, the tree-level scattering amplitude of compactification independent massless states is obtained by multiplying $\mathcal{A}$ by its right-moving counterpart and by replacing the $4 \mathrm{D} \mathrm{N}=1$ super-Yang-Mills prepotential $V(x, \theta, \bar{\theta})$ with the $4 \mathrm{D} \mathrm{N}=2$ supergravity plus tensor prepotential $U\left(x, \theta_{L}, \bar{\theta}_{L} ; \theta_{R}, \bar{\theta}_{R}\right)$. In standard $\mathrm{SU}(2)$ notation for $\mathrm{N}=2$ superspace, $\theta_{L}^{\alpha}=\theta_{+}^{\alpha}, \bar{\theta}_{L}^{\dot{\alpha}}=\bar{\theta}^{+\dot{\alpha}}, \theta_{R}^{\alpha}=\theta_{-}^{\alpha}$, and $\bar{\theta}_{R}^{\dot{\alpha}}=\bar{\theta}^{-\dot{\alpha}}$.

As discussed in reference [11], $U$ is a scalar superfield which describes an $\mathrm{N}=2$ conformal supergravity multiplet and an $\mathrm{N}=2$ tensor hypermultiplet. The NS-NS fields for the graviton, anti-symmetric tensor, and dilaton are in the $\theta_{R} \bar{\theta}_{R} \theta_{L} \bar{\theta}_{L}$ component of $U$,

$$
h_{m n}+b_{m n}+\eta_{m n} \phi=\sigma_{m}^{\alpha \dot{\alpha}} \sigma_{n}^{\beta \dot{\beta}}\left[D_{L \alpha}, \bar{D}_{L \dot{\alpha}}\right]\left[D_{R \beta}, \bar{D}_{R \dot{\beta}}\right] U,
$$

and the R-R field strengths for the $\mathrm{U}(1)$ vector and complex scalar are in the $\left(\theta_{L}\right)^{2} \bar{\theta}_{L}$ $\left(\theta_{R}\right)^{2} \bar{\theta}_{R}$ and $\theta_{L}\left(\bar{\theta}_{L}\right)^{2}\left(\theta_{R}\right)^{2} \bar{\theta}_{R}$ components of $U$,

$$
F_{m n}=\sigma_{m n}^{\dot{\alpha} \dot{\beta}}\left(D_{L}\right)^{2} \bar{D}_{L \dot{\alpha}}\left(D_{R}\right)^{2} \bar{D}_{R \dot{\beta}} U+c . c ., \quad \partial_{m} y=\sigma_{m}^{\alpha \dot{\beta}} D_{L \alpha}\left(\bar{D}_{L}\right)^{2}\left(D_{R}\right)^{2} \bar{D}_{R \dot{\beta}} U .
$$

For $U$ to be an $\mathrm{N}=2$ primary field, it must satisfy the constraints

$$
\left(D_{L}\right)^{2} U=\left(\bar{D}_{L}\right)^{2} U=\left(D_{R}\right)^{2} U=\left(\bar{D}_{R}\right)^{2} U=\partial_{m} \partial^{m} U=0 .
$$

The first four constraints are the $\mathrm{N}=2$ supersymmetric generalization of the usual polarization conditions, and the last constraint is the equation of motion in this gauge.

The topological prescription for the $\mathrm{N}=2$ closed superstring tree-level amplitude is

$$
\begin{gathered}
\mathcal{A}=<U_{1}\left(z_{1}\right) G_{R}^{+}\left(G_{L}^{+}\left(U_{2}\left(z_{2}\right)\right)\right) \tilde{G}_{R}^{+}\left(\tilde{G}_{L}^{+}\left(U_{3}\left(z_{3}\right)\right)\right) \\
\prod_{r=4}^{N} \int d z_{r} d \bar{z}_{r} G_{R}^{-}\left(G_{L}^{-}\left(G_{R}^{+}\left(G_{L}^{+}\left(U_{r}\left(z_{r}\right)\right)\right)\right)\right)>
\end{gathered}
$$

where $G_{R}^{ \pm}$and $G_{L}^{ \pm}$are the right and left-moving fermionic $\mathrm{N}=2$ generators.

Using the same methods as in the open superstring computation, one finds

$$
\begin{gathered}
\mathcal{A}=\left(\left.\prod_{r=1}^{N} \frac{\partial}{\partial \epsilon_{L r}} \frac{\partial}{\partial \epsilon_{R r}}\right|_{\epsilon_{L r}=e_{R r}=0}\right) \prod_{s=4}^{N} \int d z_{s} d \bar{z}_{s} \epsilon_{L 1} \epsilon_{R 1} \\
\int d^{2} \theta_{L 0} d^{2} \bar{\theta}_{L 0} \int d^{2} \theta_{R 0} d^{2} \bar{\theta}_{R 0}: \exp \left(F_{L}+F_{R}\right): \\
\left|z_{2}-z_{3}\right|^{4} \prod_{r, s}\left|z_{r}-z_{s}\right|^{2 k_{r}^{m} k_{s m}} \prod_{r} U_{r}\left(z_{r}\right)
\end{gathered}
$$

where $F_{L}$ is the object in the exponential of (3.4) with $k_{r}^{m}$ replaced by $\frac{1}{2} k_{r}^{m}$, and $F_{R}$ is its right-moving counterpart (i.e. $F_{R}$ is obtained from $F_{L}$ by switching $\left(\epsilon_{L r}, D_{L r}, w_{L r}, a_{L r}\right)$ to $\left(\epsilon_{R r}, D_{R r}, w_{R r}, a_{R r}\right)$ and by switching $z_{r}$ to $\left.\bar{z}_{r}\right)$. 


\section{Koba-Nielsen formula in the presence of a $D$-brane}

For closed string scattering in the presence of a D-brane, there are boundary conditions on the fields which can be either Neumann or Dirichlet. For convenience, the boundary will be chosen to be the real line, and the closed strings to reside in the upper half-plane.

The boundary conditions when $z=\bar{z}$ for the four-dimensional fields are [12]

$$
\begin{gathered}
\partial x^{m}=(-1)^{n_{m}} \bar{\partial} x^{m}, \quad \theta_{L}^{\alpha}=M_{\beta}^{\alpha} \theta_{R}^{\beta}, \quad d_{L}^{\alpha}=M_{\beta}^{\alpha} d_{R}^{\beta}, \\
\bar{\theta}_{L}^{\dot{\alpha}}=M_{\dot{\beta}}^{\dot{\alpha}} \bar{\theta}_{R}^{\dot{\beta}}, \quad \bar{d}_{L}^{\dot{\alpha}}=M_{\dot{\beta}}^{\dot{\alpha}} \bar{d}_{R}^{\dot{\beta}}, \quad \rho_{L}=\rho_{R}
\end{gathered}
$$

where $M_{\beta}^{\alpha}$ and $M_{\dot{\beta}}^{\dot{\alpha}}$ are the matrix elements of $M=\prod_{m=0}^{3} \gamma_{m}^{n_{m}}$, and $n_{m}=0$ or 1 for Neumann or Dirichlet boundary conditions in the $m^{\text {th }}$ spacetime direction. (We are assuming in this letter that $\sum_{m=0}^{3} n_{m}$ is even. When $\sum_{m=0}^{3} n_{m}$ is odd, one needs to switch $\theta_{R}^{\alpha}$ with $\bar{\theta}_{R}^{\dot{\alpha}}$ and $d_{R}^{\alpha}$ with $\bar{d}_{R}^{\dot{\alpha}}$.) Note that because the four-dimensional variables are independent of the compactification, (5.1)is independent of the boundary conditions in the compactification directions.

The first step in calculating the scattering with a D-brane is to write down the topological prescription. Comparing with the RNS prescription, [9]

$$
\begin{aligned}
\mathcal{A}= & <\xi_{L}\left(z_{1}\right) V_{1}^{R N S}\left(z_{1}\right) \int d z_{2}\left\{b_{L}, Z_{R} Z_{L} V_{2}^{R N S}\left(z_{2}\right)\right\} \\
& \prod_{r=3}^{N} \int d z_{r} d \bar{z}_{r}\left[b_{R},\left\{b_{L}, Z_{R} Z_{L} V_{r}^{R N S}\left(z_{r}\right)\right\}\right]>,
\end{aligned}
$$

and using the $\mathrm{N}=1 / \mathrm{N}=2$ relationship which is discussed after (2.2), one sees that the correct topological prescription is

$$
\begin{gathered}
\mathcal{A}=<\tilde{G}_{R}^{+}\left(U_{1}\left(z_{1}\right)\right) \int d z_{2} G_{L}^{-}\left(G_{L}^{+}\left(G_{R}^{+}\left(U_{2}\left(z_{2}\right)\right)\right)\right) \\
\prod_{r=3}^{N} \int d z_{r} d \bar{z}_{r} G_{R}^{-}\left(G_{L}^{-}\left(G_{R}^{+}\left(G_{L}^{+}\left(U_{r}\left(z_{r}\right)\right)\right)\right)\right)>
\end{gathered}
$$

Plugging in the vertex operator for the massless fields,

$$
\begin{aligned}
\mathcal{A}=( & \left.\left.\prod_{r=1}^{N} \frac{\partial}{\partial \epsilon_{L r}} \frac{\partial}{\partial \epsilon_{R r}}\right|_{\epsilon_{L r}=\epsilon_{R r}=0}\right) \int d z_{2} \prod_{s=3}^{N} \int d z_{s} d \bar{z}_{s} \epsilon_{L 1} \\
& <\exp \left(\sum _ { r } \epsilon _ { L r } \left(d_{L \alpha}\left(z_{r}\right) w_{L r}^{\alpha}+\bar{d}_{L \dot{\alpha}}\left(z_{r}\right) \bar{w}_{L r}^{\dot{\alpha}}\right.\right.
\end{aligned}
$$




$$
\begin{gathered}
\left.+\partial \theta_{L \alpha}\left(z_{r}\right) a_{L r}^{\alpha}+\partial \bar{\theta}_{L \dot{\alpha}}\left(z_{r}\right) \bar{a}_{L r}^{\dot{\alpha}}+\Pi_{L m}\left(z_{r}\right) a_{L r}^{m}\right) \\
\exp \left(\sum _ { r } \epsilon _ { R r } \left(d_{R \alpha}\left(\bar{z}_{r}\right) w_{R r}^{\alpha}+\bar{d}_{R \dot{\alpha}}\left(\bar{z}_{r}\right) \bar{w}_{R r}^{\dot{\alpha}}\right.\right. \\
\left.+\bar{\partial} \theta_{R \alpha}\left(\bar{z}_{r}\right) a_{R r}^{\alpha}+\partial \bar{\theta}_{R \dot{\alpha}}\left(z_{r}\right) \bar{a}_{R r}^{\dot{\alpha}}+\Pi_{R m}\left(\bar{z}_{r}\right) a_{R r}^{m}\right) \\
e^{-2 i \rho_{R}\left(\bar{z}_{1}\right)+i H_{R C}\left(\bar{z}_{1}\right)} e^{i \rho_{R}\left(\bar{z}_{2}\right)} \prod_{r=1}^{N} U_{r}\left(z_{r}\right)>
\end{gathered}
$$

where the only non-zero $w$ 's and $a$ 's are

$$
\bar{w}_{r}^{\dot{\alpha}}=\left(D_{r}\right)^{2} \bar{D}_{r}^{\dot{\alpha}}, w_{r}^{\alpha}=\left(\bar{D}_{r}\right)^{2} D_{r}^{\alpha}, a_{r}^{\alpha}=D_{r}^{\alpha}, \bar{a}_{r}^{\dot{\alpha}}=-\bar{D}_{r}^{\dot{\alpha}}, a_{r}^{\alpha \dot{\alpha}}=-i\left[D_{r}^{\alpha}, \bar{D}_{r}^{\dot{\alpha}}\right]
$$

for $r>1$ when left-moving and for $r>2$ when right-moving, and

$$
\bar{w}_{R 1}^{\dot{\alpha}}=\bar{D}_{R 1}^{\dot{\alpha}}, w_{R 2}^{\alpha}=D_{R 2}^{\alpha}
$$

One can now use the techniques of section 3 to compute the correlation function. The only difference is that the correlation function of left-moving fields must equal the correlation function of its right-moving partner when the field sits on the boundary. For example,

$$
<d_{L}^{\alpha}(z) \ldots>=<\sum_{s} \frac{f_{L s}^{\alpha}\left(z_{s}\right)}{z-z_{s}}+\sum_{t} \frac{f_{R t}^{\alpha}\left(z_{t}\right)}{z-\bar{z}_{t}}>
$$

where $f_{L s}^{\alpha}$ is the pole residue of $d_{L}^{\alpha}(z)$ at $z=z_{s}$ and $f_{R t}^{\alpha}$ is the pole residue of $M_{\beta}^{\alpha} d_{R}^{\beta}(z)$ at $\bar{z}=\bar{z}_{t}$.

The resulting Koba-Nielsen formula in the presence of a D-brane is:

$$
\begin{aligned}
& \mathcal{A}=\left(\left.\prod_{r=1}^{N} \frac{\partial}{\partial \epsilon_{L r}} \frac{\partial}{\partial \epsilon_{R r}}\right|_{\epsilon_{L r}=e_{R r}=0}\right) \int d z_{2} \prod_{s=3}^{N} \int d z_{s} d \bar{z}_{s} \epsilon_{L 1} \int d^{2} \theta_{0} d^{2} \bar{\theta}_{0} \\
&: \exp \left[\left[\sum_{r, s} \epsilon_{L r}\left(\frac{D_{L s \alpha} w_{L r}^{\alpha}+\bar{D}_{L s \dot{\alpha}} \bar{w}_{L r}^{\dot{\alpha}}+k_{s m} a_{L r}^{m}}{z_{r}-z_{s}}+\epsilon_{L s} \frac{a_{L s \alpha} w_{L r}^{\alpha}+\bar{a}_{L s \dot{\alpha}} \bar{w}_{L r}^{\dot{\alpha}}}{\left(z_{r}-z_{s}\right)^{2}}\right)\right.\right. \\
&+i\left[\sum_{r, s, t} \epsilon_{L r} \epsilon_{L s}\left(\frac{w_{L r}^{\alpha} \bar{w}_{L s}^{\dot{\alpha}} k_{t \alpha \dot{\alpha}}}{\left(z_{s}-z_{r}\right)\left(z_{s}-z_{t}\right)}+\epsilon_{L t} \frac{w_{L r}^{\alpha} \bar{w}_{L s}^{\dot{\alpha}} a_{L t \alpha \dot{\alpha}}}{\left(z_{s}-z_{t}\right)\left(z_{r}-z_{t}\right)\left(z_{r}-z_{s}\right)}\right)\right. \\
& \quad+\frac{1}{4}\left[\sum_{r, s, t, u} \epsilon_{L r} \epsilon_{L s} \epsilon_{L t} \epsilon_{L u} \frac{\bar{w}_{L s \dot{\alpha}} \bar{w}_{L u}^{\dot{\alpha}} w_{L t \alpha} w_{L r}^{\alpha}}{\left(z_{r}-z_{s}\right)\left(z_{t}-z_{u}\right)\left(z_{r}-z_{u}\right)\left(z_{t}-z_{s}\right)}\right. \\
&+\{L u \rightarrow R u\}]+\{L t \rightarrow R t\}]+\{L s \rightarrow R s\}]+\{L r \rightarrow R r\}]
\end{aligned}
$$




$$
\left(\bar{z}_{1}-\bar{z}_{2}\right)^{2} \prod_{r, s}\left|z_{r}-z_{s}\right|^{2 k_{r}^{m} k_{s m}}\left|z_{r}-\bar{z}_{s}\right|^{2 k_{r}^{m} \hat{k}_{s m}} \prod_{r} U_{r}\left(z_{r}\right)
$$

where $\hat{k}_{r}^{m}=(-1)^{n_{m}} k_{r}^{m}, \int d^{2} \theta_{0} d^{2} \bar{\theta}_{0}$ means to set $\theta_{L}^{\alpha}\left(z_{r}\right)=M_{\beta}^{\alpha} \theta_{R}^{\beta}\left(\bar{z}_{r}\right)=\theta_{0}^{\alpha}$ and $\bar{\theta}_{L}^{\dot{\alpha}}\left(z_{r}\right)=$ $M_{\dot{\beta}}^{\dot{\alpha}} \bar{\theta}_{R}^{\dot{\beta}}\left(\bar{z}_{r}\right)=\bar{\theta}_{0}^{\dot{\alpha}}$ for all $r$ and to integrate over $\theta_{0}^{\alpha}$ and $\bar{\theta}_{0}^{\dot{\alpha}}$, and $\{L u \rightarrow R u\}$ means to switch $z_{u} \rightarrow \bar{z}_{u}$ and to switch

$$
\begin{gathered}
\left(\epsilon_{L u}, w_{L u}^{\alpha}, \bar{w}_{L u}^{\dot{\alpha}}, a_{L u}^{\alpha}, a_{L u}^{\dot{\alpha}}, a_{L u}^{m}, k_{u}^{m}\right) \rightarrow \\
\left(\epsilon_{R u}, M_{\beta}^{\alpha} w_{R u}^{\beta}, M_{\dot{\beta}}^{\dot{\alpha}} \bar{w}_{L u}^{\dot{\beta}}, M_{\beta}^{\alpha} a_{L u}^{\alpha}, M_{\dot{\beta}}^{\dot{\alpha}} a_{L u}^{\dot{\beta}},(-1)^{n_{m}} a_{L u}^{m}, \hat{k}_{u}^{m}\right) .
\end{gathered}
$$

Note that the switch affects everything inside the [] brackets, e.g. $\{L u \rightarrow R u\}$ affects only the $\epsilon_{r} \epsilon_{s} \epsilon_{t} \epsilon_{u}$ term but $\{L r \rightarrow R r\}$ affects all terms (including those coming from earlier switches).

In summary, the manifestly super-Poincaré invariant Koba-Nielsen formulas for massless tree amplitudes of the open superstring, closed superstring, and closed superstring in the presence of a D-brane can be found in equations (3.4), (4.1), and (5.5).

Acknowledgements: I would like to thank Vipul Periwal and Øjvind Tafjord for suggesting the D-brane generalization and for sharing their results using light-cone GreenSchwarz calculations. 


\section{References}

[1] M.B. Green and J.H. Schwarz, Nucl. Phys. B243 (1984) p.475.

[2] S. Mandelstam, Prog. Theor. Phys. Suppl. 86 (1986) p.163.

[3] A. Restuccia and J.G. Taylor, Phys. Rep. 174 (1989) p.283.

[4] S. Mandelstam, Workshop on Unified String Theories, 29 July - 16 August 1985, eds. M. Green and D. Gross, World Scientific, Singapore (1986) p. 577.

[5] N. Berkovits, Nucl. Phys. B431 (1994) p.258.

[6] N. Berkovits, Nucl. Phys. B420 (1994) p.332;

N. Berkovits and C. Vafa, Mod. Phys. Lett. A9 (1994) p.653.

[7] N. Berkovits and C. Vafa, Nucl. Phys. B433 (1995) p.123.

[8] N. Berkovits, "A New Description of the Superstring", preprint IFUSP-P-1212, April 1996, to appear on hep-th.

[9] J. Polchinski, "Dirichlet-Branes and Ramond-Ramond Charges", hep-th 9510017;

S.S. Gubser, A. Hashimoto, I.R. Klebanov and J.M. Maldacena, "Gravitational Lensing by $p$-Branes", hep-th 9601057 ;

M.R. Garousi and R.C. Meyers, "Superstring Scattering from D-Branes", hep-th 9603194 .

[10] W. Siegel, Nucl. Phys. B263 (1986) p.93.

[11] N. Berkovits and W. Siegel, "Superspace effective actions for 4D compactifications of heterotic and type II superstrings", preprint IFUSP-P-1180, October 1995, to appear in Nucl. Phys. B, hep-th 9501016.

[12] V. Periwal and $\varnothing$. Tafjord, private communication;

M.B. Green, Phys. Lett. B329 (1994), p. 435. 